\title{
A NONLINEAR GENERALIZATION OF BIHARI'S INEQUALITY
}

\author{
U. D. DHONGADE AND S. G. DEO
}

\begin{abstract}
The aim of the present note is to prove a nonlinear generalization of the well-known integral inequality due to Bihari. This generalization is useful in obtaining pointwise estimates of solutions of nonlinear Volterra integral equations.
\end{abstract}

1. Introduction. It is widely recognized today, that the integral inequalities furnish a very general comparison principle not only in the study of stability but in studying many other qualitative as well as quantitative properties of solutions of differential equations. The well-known Gronwall inequality and its generalization due to Bihari [1] have been frequently employed in this direction. Such types of inequalities are profitably used by Brauer [2] to study the asymptotic behaviour of the solutions of differential systems. Recently [3], [4] linear and nonlinear generalizations of these inequalities have been obtained while studying the pointwise estimates of linear and nonlinear Volterra integral equations. Analogous to the linear generalization of Gronwall's inequality due to Willett [5] we aim in obtaining a nonlinear generalization of Bihari's inequality under suitable conditions and illustrates its usefulness.

2. Preliminaries. In the sequel, let $I$ denote the set of positive real numbers $0<x<\infty$ and $C_{0}$ the class of continuous functions defined on $I$. Further we need the class of functions $\mathscr{F}$ defined below.

Definition. A function $\Phi$ is said to belong to a class $\mathscr{F}$ if it satisfies the following conditions:

(i) $\Phi(u)>0$ is nondecreasing and $\Phi \in C_{0}$ for $u>0$,

(ii) $(1 / v) \Phi(u) \leqslant w(u / v)$ for all $u$ and $v \geqslant 1$, where $w$ is a positive, nondecreasing function defined and continuous on $I$. This class of functions has been widely employed in Theorem 9 [1]. One of the functions belonging to $\mathscr{F}$ is the function $\Phi$ defined as $\Phi(u)=\sum_{i=1}^{n} u^{\alpha_{i}}, \alpha_{i} \leqslant 1, i=1,2,3, \ldots, n$. The main theorem is based on the following lemma.

Lemma. Suppose

(i) $g(x), h(x) \in C_{0}$ and $g(x) \geqslant 1$,

(ii) $\Phi \in \mathcal{F}$,

(iii) $f>0$ is nondecreasing on $I, f \in C_{0}$, and

$$
y(x) \leqslant f(x)+g(x) \int_{0}^{x} h(s) \Phi(y(s)) d s, \quad x \in I .
$$

Received by the editors December 19, 1973 and, in revised form, December 28, 1974. AMS (MOS) subject classifications (1970). Primary 34A40; Secondary 45D05.

Key words and phrases. Bihari's inequality, Volterra integral equations, Gronwall inequality. 
Then

(2)

$$
y(x) \leqslant f(x) g(x) G^{-1}\left[G(1)+\int_{0}^{x} h(s) g(s) d s\right], \quad 0<x \leqslant b,
$$

where

$$
G(u)=\int_{u_{0}}^{u} \frac{d s}{w(s)}, \quad 0 \leqslant u_{0}, u \geqslant 0,
$$

and $G^{-1}$ is the inverse of $G$ and $x$ is in the subinterval $(0, b]$ of $I$ so that

$$
G(1)+\int_{0}^{x} h(s) g(s) d s \in \operatorname{Dom}\left(G^{-1}\right) \text {. }
$$

Proof. Since $f(x)$ is nondecreasing, $\Phi \in \mathscr{F}$ and $g(x) \geqslant 1$, we have from (1)

$$
\begin{aligned}
\frac{y(x)}{f(x)} & \leqslant 1+g(x) \int_{0}^{x} \frac{h(s) \Phi(y(s))}{f(s)} d s \\
& \leqslant g(x)\left[1+\int_{0}^{x} \frac{h(s) \Phi(y(s))}{f(s)} d s\right] .
\end{aligned}
$$

Hence

$$
\frac{y(x)}{f(x) g(x)} \leqslant 1+\int_{0}^{x} h(s) g(s) w\left(\frac{y(s)}{f(s) g(s)}\right) d s .
$$

Now we apply the integral inequality due to Bihari [1] to obtain the estimate given in (2). If $g(x) \equiv 1$ in (1) then Theorem 3 proved in [3] follows.

3. Main result. It is natural to think of a nonlinear generalization of Bihari's integral inequality analogous to that considered by Willett [5]. The following theorem, proved under some conditions answers this question.

THEOREM 1. Suppose

(i) $f(x)$ be defined as in the lemma,

(ii) $h_{i}(x) \in C_{0}$ for $i=1,2, \ldots, n$,

(iii) $\Phi_{i} \in \mathcal{F}, i=1,2, \ldots, n$,

and the following integral inequality holds

$$
y(x) \leqslant f(x)+\sum_{i=1}^{n} \int_{0}^{x} h_{i}(s) \Phi_{i}(y(s)) d s, \quad x \in I .
$$

Then

$$
y(x) \leqslant f(x) \prod_{i=1}^{n} E_{i}(x), \quad 0<x \leqslant b, 0<b<\infty,
$$

where the functions $G_{k}$ are defined as

$$
G_{k}(u)=\int_{u_{0}}^{u} \frac{d s}{w_{k}(s)}, \quad 0<u_{0}, u \geqslant 0
$$

and 


$$
\begin{aligned}
& E_{1}(x)=G_{1}^{-1}\left[G_{1}(1)+\int_{0}^{x} h_{1}(s) d s\right], \\
& E_{k}(x)=G_{k}^{-1}\left[G_{k}(1)+\int_{0}^{x} h_{k}(s) \prod_{i=1}^{k-1} E_{i}(s) d s\right], \quad k=2,3, \ldots, n,
\end{aligned}
$$

$G_{k}^{-1}$ being the inverse of $G_{k}$ and $x$ is in the subinterval $(0, b]$ of $I$ so that

$$
G_{1}(1)+\int_{0}^{x} h_{1}(s) d s \in \operatorname{Dom}\left(G_{1}^{-1}\right)
$$

and

$$
G_{k}(1)+\int_{0}^{x} h_{k}(s) \prod_{i=1}^{k-1} E_{i}(s) d s \in \operatorname{Dom}\left(G_{k}^{-1}\right), \quad k=2,3, \ldots, n .
$$

Proof. The proof is by mathematical induction. Note that for $n=1$ the Theorem 1 is a special case of the lemma and hence is true. Let us assume that (5) is true for some integer $k, 1<k \leqslant n-1$, that is

$$
y(x) \leqslant f(x) \prod_{i=1}^{k} E_{i}(x)=f(x) R(x) \quad \text { (say) }
$$

where $R(x)=\prod_{i=1}^{k} E_{i}(x)$. Hence

$$
y(x) \leqslant R(x)\left[f(x)+\int_{0}^{x} h_{k+1}(s) \Phi_{k+1}(y(s)) d s\right] .
$$

Now in view of the assumption on $f$ and $\Phi_{i}$ we obtain

$$
\frac{y(x)}{f(x) R(x)} \leqslant 1+\int_{0}^{x} h_{k+1}(s) R(s) w_{k+1}\left(\frac{y(s)}{f(s) R(s)}\right) d s .
$$

This inequality is of the form (4) and hence by the lemma, proved earlier, we have

$$
\begin{aligned}
y(x) \leqslant f(x) R(x) G_{k+1}^{-1}\left[G_{k+1}(1)+\int_{0}^{x} h_{k+1}(s) R(s) d s\right], & \\
0 & <x<b<\infty .
\end{aligned}
$$

Substituting the value of $R(x)$ and using (6) it is easy to write (7) as

$$
y(x) \leqslant f(x) \prod_{i=1}^{k+1} E_{i}(x) .
$$

This proves that (5) is true for $k+1$. The proof is complete.

ExAmple. The following example illustrates Theorem 1. Let

$$
y(x) \leqslant f(x)+\sum_{i=1}^{3} \int_{0}^{x} h_{i}(s) y^{\alpha_{i}}(s) d s,
$$

where $\alpha_{i}<1, i=1,2,3$. We note that $y^{\alpha_{i}} \in \bar{y}$ on $I$ and $f, h_{i}(i=1,2,3)$ are as defined in Theorem 1. It is easy to observe

$$
G_{i}(u)=\frac{1}{1-\alpha_{i}}\left[u^{1-\alpha_{i}}-u_{0}^{1-\alpha_{i}}\right], \quad i=1,2,3,
$$

and 


$$
G_{i}^{-1}(u)=\left[\left(1-\alpha_{i}\right) u+u_{0}^{1-\alpha_{i}}\right]^{1 /\left(1-\alpha_{i}\right)}, \quad i=1,2,3 .
$$

Similarly we obtain

$$
\begin{aligned}
& E_{1}(x)=\left[1+\left(1-\alpha_{1}\right) \int_{0}^{x} h_{1}(s) d s\right]^{1 /\left(1-\alpha_{1}\right)}, \\
& E_{2}(x)=\left[1+\left(1-\alpha_{2}\right) \int_{0}^{x} h_{2}(s) E_{1}(s) d s\right]^{1 /\left(1-\alpha_{2}\right)}, \\
& E_{3}(x)=\left[1+\left(1-\alpha_{3}\right) \int_{0}^{x} h_{3}(s) E_{2}(s) d s\right]^{1 /\left(1-\alpha_{3}\right)} .
\end{aligned}
$$

We conclude that $y(x) \leqslant f(x) \prod_{i=1}^{3} E_{i}(x)$.

4. Applications. Consider a nonlinear Volterra integral equation

$$
y(x)=f(x)+\int_{0}^{x} k(x, s) \Phi(y(s)) d s,
$$

where $k(x, s)$ is a nonnegative function and the function $\Phi$ is as given in the lemma. We show that the above theorem can be profitably employed to obtain the pointwise estimates of the solutions of the equation (8). While considering nonlinear integral equations, generally linearization techniques are exploited, however below we attempt to obtain a genuine upper bound for the solution of the equation (8) by using the inequality proved in Theorem 1.

THEOREM 2. Suppose

(i) $f(x)$ and $\Phi(u)$ be defined as in the lemma and $\Phi$ be subadditive,

(ii) $k(x, s)(x \geqslant s)$ be defined and continuous on $I \times I$,

(iii) $k(x, x) \leqslant h_{1}(x),(\partial k / \partial x)(x, s) \leqslant g(x) h_{2}(s)$,

where $g(x), h_{1}(s), h_{2}(s)$ are continuous on $I$ and $y(x)$ satisfies $(8)$ on I. Then

$$
|y(x)| \leqslant f(x)+p(x) E_{1}(x) E_{2}(x)
$$

where

$$
\begin{aligned}
& E_{1}(x)=G^{-1}\left[G(1)+\int_{0}^{x} h_{1}(s) d s\right] \\
& E_{2}(x)=G^{-1}\left[G(1)+\int_{0}^{x} h_{2}(s) m(s) E_{1}(s) d s\right] \\
& p(x)=\int_{0}^{x} h_{1}(s) \Phi(f(s)) d s+m(x) \int_{0}^{x} h_{2}(s) \Phi(f(s)) d s
\end{aligned}
$$

and

$$
m(x)=\int_{0}^{x} g(s) d s .
$$

$G^{-1}$ being the inverse of $G$, which is defined as in the lemma, and $x$ is so that

$$
G(1)+\int_{0}^{x} h_{1}(s) d s \in \operatorname{Dom}\left(G^{-1}\right)
$$

and

$$
G(1)+\int_{0}^{x} h_{2}(s) m(s) E_{1}(s) d s \in \operatorname{Dom}\left(G^{-1}\right)
$$


Proof. Since $y(x)$ satisfies (8) we have

$$
|y(x)| \leqslant f(x)+\int_{0}^{x} k(x, s) \Phi(|y(s)|) d s .
$$

Define

$$
R(x)=\int_{0}^{x} k(x, s) \Phi(|y(s)|) d s, \quad x \in I,
$$

then it is easy to note that

$$
R^{\prime}(x)=k(x, x) \Phi(|y(x)|)+\int_{0}^{x} \frac{\partial k(x, s)}{\partial x} \Phi(|y(s)|) d s
$$

Further using the subadditivity property of $\Phi$ and condition (iii) we obtain

$$
\begin{aligned}
R^{\prime}(x) \leqslant & h_{1}(x) \Phi(f(x))+g(x) \int_{0}^{x} h_{2}(s) \Phi(f(s)) d s \\
& +h_{2}(x) \Phi(R(x))+g(x) \int_{0}^{x} h_{2}(s) \Phi(R(s)) d s .
\end{aligned}
$$

Integrating from 0 to $x$, we get

$$
\begin{aligned}
R(x) \leqslant & \int_{0}^{x} h_{1}(s) \Phi(f(s)) d s+\int_{0}^{x} g(s)\left(\int_{0}^{s} h_{2}(t) \Phi(f(t)) d t\right) d s \\
& +\int_{0}^{x} h_{1}(s) \Phi(R(s)) d s+\int_{0}^{x} g(s)\left(\int_{0}^{s} h_{2}(t) \Phi(R(t)) d t\right) d s .
\end{aligned}
$$

Now replacing the limits ' $s$ ' by ' $x$ ' the inequality still holds and becomes the product of two integrals.

In view of the definitions of $m(x)$ and $p(x)$ one can rewrite this inequality as

$$
R(x) \leqslant p(x)+\int_{0}^{x} h_{1}(s) \Phi(R(s)) d s+m(x) \int_{0}^{x} h_{2}(s) \Phi(R(s)) d s .
$$

By applying Theorem 1 for $n=2$, we obtain

$$
R(x) \leqslant p(x) E_{1}(x) E_{2}(x) .
$$

On substituting this bound in (10) the estimate (9) is obtained. For more general applications, one may replace the condition (iii) by

$$
\frac{\partial k(x, s)}{\partial x} \leqslant \sum_{i=1}^{n} g_{i}(x) h_{i}(s) \text {. }
$$

We obtain below, as an illustration, pointwise estimates of the solution of the nonlinear integral equation (8) when $f(x)=x^{2}, k(x, s)=e^{x}\left(1 /\left(1+e^{s}\right)\right)^{2}$ and $\Phi(y)=\sqrt{y}$. Note that $f$ satisfies the condition in Theorem $2, \Phi \in \mathscr{F}$ and is subadditive. Further

$$
\begin{aligned}
k(x, x) & =e^{x}\left(\frac{1}{1+e^{x}}\right)^{2}<e^{x}=h_{1}(x) \quad \text { (say), } \\
\frac{\partial k(x, s)}{\partial x} & =e^{x}\left(\frac{1}{1+e^{s}}\right)^{2}<e^{x} \cdot e^{-2 s} .
\end{aligned}
$$


Assume that

$$
\begin{gathered}
g(x)=e^{x}, \quad h_{2}(s)=e^{-2 s} \\
G(u)=2\left[\sqrt{u}-\sqrt{u_{0}}\right], \quad G^{-1}(u)=\frac{\left[u+2 \sqrt{u_{0}}\right]^{2}}{4}, \\
E_{1}(x)=\frac{\left(1+e^{x}\right)^{2}}{4}, \quad E_{2}(x)=\frac{\left(e^{x}-x+7\right)^{2}}{64}
\end{gathered}
$$

and

$$
p(x)=x e^{x}+\frac{1}{2} e^{-2 x}\left(1-e^{x}\right)\left[3 e^{2 x}+x+1\right] .
$$

Substituting these values in (9) the bound of $y(x)$ is computed.

\section{REFERENCES}

1. I. Bihari, Researches of the boundedness and stability of the solutions of non-linear differential equations, Acta. Math. Acad. Sci. Hungar. 8(1957), 261-278. MR20 \# 1031.

2. F. Brauer, Perturbations of nonlinear systems of differential equations, J. Math. Anal. Appl. 14(1966), 198-206. MR 33 \#359.

3. U. D. Dhongade and S. G. Deo, Some generalizations of Bellman-Bihari integral inequalities, J. Math. Anal. Appl. 44(1973), 218-226.

4. , Pointwise estimates of solutions of some Volterra integral equations, J. Math. Anal. Appl. 45(1974), 615-628.

5. D. W. Willett, A linear generalization of Gronwall's inequality, Proc. Amer. Math. Soc. 16(1965), 774-778. MR31 \#5953.

Department of Mathematics, S. B. College, Aurangabad, Maharashtra, India

Department of Mathematics, Centre for Post-Graduate Instruction and Research, PANAJi, GoA, INDIA 